\title{
The Exploitation of Attenuation Measurements for Atmosphere Monitoring
}

\author{
Dino Giuli, Simone Tanelli, Luca Facheris, Fabrizio Cuccoli, M. Gherardelli \\ Dipartimento di Elettronica e Telecomunicazioni - Università di Firenze, Via di S. Marta, 350139 Firenze - Italy \\ Tel: +390554796370 Fax: +39055488883 E-mail: giuli@diefi.die.unifi.it
}

\begin{abstract}
This paper is an overview of research contributions by the Department of Electronics and Telecommunications of the University of Florence to the use of attenuation measurements for monitoring the atmosphere. The objective is to point out the potential of this kind of measurements in different contexts of ground and spaceborne remote sensing, and to stimulate new research exploitations.
\end{abstract}

\section{INTRODUCTION}

Remote sensing of the atmosphere can exploit appropriate use of path-integrated attenuation measurements based on a single or multiple transmitter-receiver links. In this paper we point out how such kind of attenuation measurements can be utilized in extremely different applications, exploiting the geometry of multiple measurement links and/or the joint use of several frequencies. The great availability of spaceborne/airborne transport systems and of ground communication systems, allowing a high number of possible configurations (number of links and geometry, horizontal or vertical displacement, etc.), provides a wide range of applicability fields of atmospheric remote sensing. The attenuation information obtained by such systems requires ad hoc data processing procedures, that may vary with the application. For instance, if the objective is to retrieve 2D fields, use can be made of tomographic data processing applied to several 1D average measurements. In this regard notice however that conventional tomographic inversion procedures cannot be used: in fact, for evident reasons of costs and logistics, in remote sensing applications the relatively low number of links and their displacement geometry are not adequate to apply standard inversion methods. The main consequence is that the inversion problem is highly ill-conditioned: to get an idea of two different possible approaches to face such ill-conditioning, the reader can refer to [1].

A relevant problem that comes prior to the optimal inversion methods is a preliminary feasibility study. This is in general needed for each application: its complexity depends on the frequency range and on the number of frequencies utilized for attenuation measurements, and on the cause of such attenuation (i.e., if it is due to the joint effect of scattering and absorption properties of the propagation medium, or if one of them dominates).

In the paper are first recalled two application examples, concerning rainfall and water vapor monitoring. Though measurements are made at microwaves in both cases, they differ substantially (for the need of a more complex feasibility study and for the use of multifrequency in the second case). After that, we emphasize that analogous methods can be applied also in the infrared spectral regions, where attenuation measurements can be exploited for monitoring pollutants and atmospheric components in general, both in limited areas at ground and on more extended vertical sections of the atmosphere.

\section{MONITORING RAINFALL AT GROUND}

This first example of monitoring is described in detail in [1]. The objective is estimating the 2D distribution of rainfall intensity at ground by means of a combination of pathaveraged attenuation measurements. Such measurements refer to single frequency transmitter-receiver links (3-10) forming a microwave tomographic network over the area (about 400 $\mathrm{km}^{2}$ ) to be monitored. In this case, attenuation caused by the rainfall medium depends both on scattering and absorption properties of raindrops, and on their drop size distribution. If frequencies are avoided where dominates energy absorption by gases (mainly water vapor and oxygen), assuming a plane horizontal surface and a $x-y$ Cartesian coordinate system, the specific attenuation field $k(x, y, t)$ at time $t$ in every point $(x, y)$ is simply related to rainfall rate $R(x, y, t)$ by empirical power law relationships of the kind $k=a R^{b}$. Such relationships are particularly accurate around $35 \mathrm{GHz}$, where $a=0.221$ and $b=1.04$ with an average error smaller than $10 \%$. The feasibility study in this case is not particularly complex, involving mainly the analysis of technical system requirements and error sources [2].

Therefore, the main issue is the efficiency of the tomographic inversion algorithm (with respect both to the illconditioning and to the retrieval speed). In [1], the reconstructed rainfall field is expressed as a linear combination of $\mathrm{N}$ Gaussian cells: the unknowns are, besides $\mathrm{N}$, the four parameters of each cell (center coordinates, height and width). The inversion approach adopted is stochastic: in a first phase (generation), the space of solutions is sampled randomly, following a stochastic tree search criterion. At the end of this somewhat "blind" search, a set of random solutions is found that minimizes a predefined error functional. In a second iterative phase (optimization), the solutions are refined by searching for local minima of the error functional and for the possible physical anomalies of the current random solutions, in order to remove them. At the end of the process, a set is found of "equivalent" solutions in terms both of error functional and of their physical plausibility. This approach in two phases is particularly 
convenient in remote sensing of rainfall, where it is important to gain processing efficiency for real time tracking of rainfall cells. Given a sequence of attenuation vectors, and based on the space-time correlation of the rainfall process, it is not necessary to apply the whole tomographic algorithm to reconstruct each $2 \mathrm{D}$ snapshot. Indeed, the optimization phase only can be used, by applying it to the $2 \mathrm{D}$ reconstruction at the previous time step: when temporal decorrelation makes such starting initial condition unreliable, the method developed exhibits a characteristic behavior, indicating that the whole procedure has to be applied.

The relative simplicity of the tomographic system for rainfall monitoring makes it an alternative, or integrative solution with respect to weather radar in areas of limited size and/or surrounded by complex orography. Furthermore, it can provide an improved space-time resolution with respect to raingauge networks, at the parity of total number of measurement stations (transmitters and receivers, or raingauges) [2]. Also, it can be exploited not only as a standalone system, but also as a reference measurement system at ground in experiments for calibration of radar rainfall estimates, or for determining reliable methods to convert radar reflectivity data to rainfall estimates, since it allows short-time and area-averaged comparisons that low-density raingauge networks are not able to provide.

\section{MONITORING WATER VAPOR PROFILES}

Retrieval of vertical profiles of water vapor is typically performed by multi-frequency radiometers. However, they are subject to relevant uncertainties about the emissivity function and also suffer from a basic spatial resolution problem, related to their standard field of view. Also, the large bandwidth required to increase the received power of radiometers and their sensitivity does not allow a sufficiently fine spectral resolution as would be required to determine the shape of the vertical profiles with the precision needed in applications requiring the in depth analysis of local atmospheric conditions.

For this reason, confident that attenuation measurements based on transmitter-receiver links at microwaves can provide interesting, highly localized and more reliable information about vertical shape of water vapor concentration, our group started recently to carry out research in this field, based on a multifrequency spaceborne system approach. The first objective posed, as reported in detail in [3], consists in evaluating how the variations of water vapor profiles' shape influence the ground-satellite spectral attenuation around $22.235 \mathrm{GHz}$. Therefore, $\mathbf{a}$ in depth analysis has been carried in order to:

- quantitatively evaluate the influence of the variations of vertical profiles of temperature and pressure;
- qualitatively determine, by means of a spectral sensitivity function, the information carried by multifrequency attenuation measurements

An example of this kind of analysis is reported in Fig. 1, where the spectral sensitivity function (bottom) is reported together with the error profile (top right), considering a retrieved profile as compared to the corresponding true one (top left). The results of such investigations can be usefully exploited in order to quantitatively correct for errors in the estimates of vertical water vapor profile. In fact, a constant correlation was found between the error profiles and the spectral sensitivity function. In particular, amplitude and sign of the error profile at lower altitude (below about 2-3 km), at intermediate altitude (between $2-3$ and $7-10 \mathrm{~km}$ ), and at higher altitude (over 7-10 km) are correlated to amplitude and sign of the spectral sensitivity function respectively at lower (up to $20 \mathrm{GHz}$ ), intermediate $(20-21 \mathrm{GHz}$ ) and higher frequencies (over 20-21 GHz).
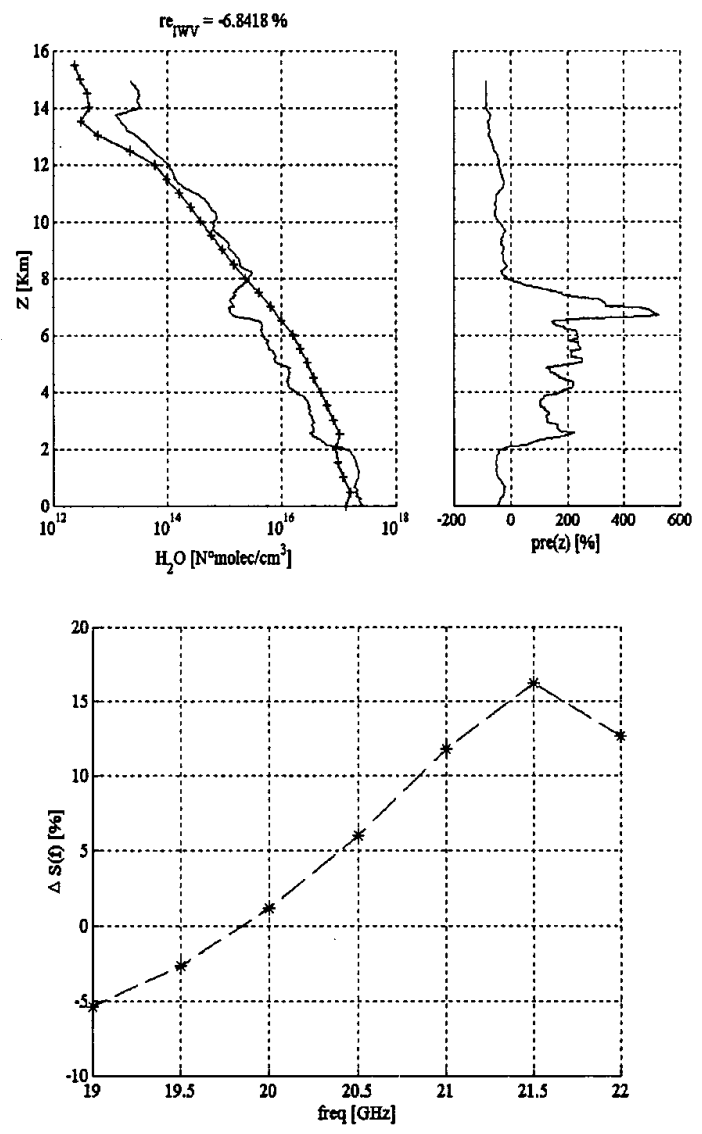

Fig. 1 Top left: Water vapor's vertical profiles. Continuous line: radiosonde profile (from experimental data); cross line: retrieved profile. (top right) percentage profile relative error. (bottom) Spectral sensitivity function computed from 19 to $22 \mathrm{GHz}$ step $0.5 \mathrm{GHz}$. 
The analysis carried out up to now has neglected clouds and rainfall effects, which evidently modify remarkably the attenuation measurements. In case clouds effects only are dominant, a tomographic approach could be desirable in order to retrieve the $2 \mathrm{D}$ vertical distribution of the cloud water content. Also, since at microwaves sufficiently high power can be emitted also by spaceborne transmitters, non directive antennas can be used to scan a sufficiently wide area with a single transmitter-receiver pair, without the need for accurate antenna pointing systems.

Availability of frequency hopping systems gives the possibility to gather $2 \mathrm{D}$ information of water vapor content in atmospheric layers through frequency analysis, before the following exploitation of any tomographic approach. Moreover, the Doppler effect generated by satellite motion could also be exploited for fine spectral analysis (sensitivity function measurements).

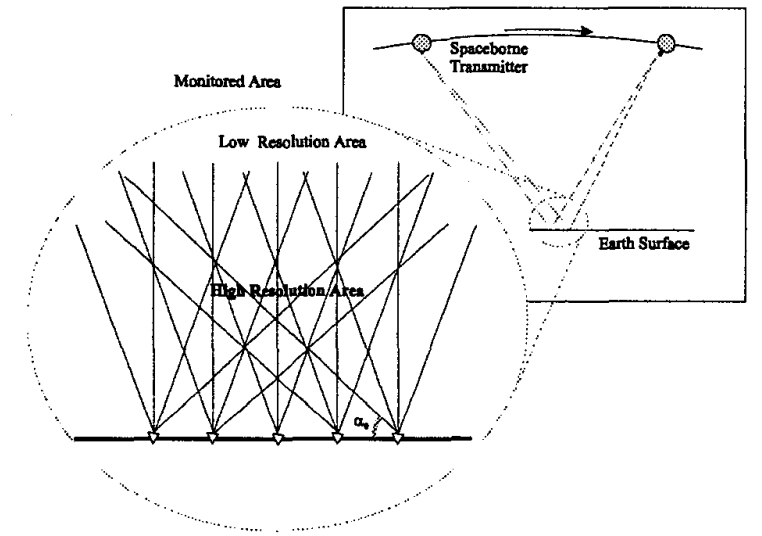

Fig. 2 General scheme of a satellite tomographic monitoring system

\section{MONITORING ATMOSPHERIC COMPONENTS}

The same tomographic inversion algorithm utilized for processing attenuation vector data for rainfall monitoring at ground can be exploited for retrieving 2D distributions of atmospheric components and pollutants in small areas (not larger than $4 \mathrm{~km}^{2}$ ) over plane surfaces parallel to the Earth. Infrared attenuation measurements can be in fact exploited to estimate, along each link, the concentration of the species of interest. Obviously, the problem in this case is complicated by the need to separate the attenuation contribution of the molecular species of interest from the total path-integrated attenuation. This is indeed a problem, but -if solved- also a remarkable measurement potential: in fact, if multifrequency measurements are made, it is possible to determine the 2D concentration of each species of interest with a single multifrequency system. Therefore, in this case, a in-depth feasibility study had to be done, concerning both the infrared system link design and a detailed analysis for the optimal choice of wavelengths at which the path averaged concentration of each species of interest can be extracted through standard spectral methods. The details of such procedures can be found in [4] in the case of the derivative method.

Also the approach used for water vapor monitoring at microwaves can be extended to infrared wavelengths. Infrared In fact, also at infrared absorption properties of atmospheric components also vary with height. In line of principle, therefore, a multiwavelength approach can be used also at infrared in order to derive information about the vertical profiles of such components. At the current time, our group has investigating a differential (dual-wavelength) method for estimating mean concentrations of $\mathrm{CO}$ and $\mathrm{CO}_{2}$ along a vertical path [5]. Such method can be extended to other atmospheric components.

It has to be noticed, however, that differently from the microwave case, a vertical tomographic approach is indeed much more complicated due to basic limitations of the utilizable infrared transmitters (viz. power, beamwidth).

\section{CONCLUSIONS}

The potential of attenuation measurements for applications involving the remote sensing of clouds and precipitation and, in general, of the atmosphere has not yet been well investigated and exploited. The purpose of this brief survey was to stimulate research on this topic.

\section{ACKNOWLEDGMENTS}

This work was supported by the Italian Space Agency and by CNR-GNDCI.

\section{REFERENCES}

[1] D. Giuli, L. Facheris, S. Tanelli "Microwave inversion technique based on stochastic approach for rainfall field monitoring" IEEE Trans. Geosc. Rem. Sens. 37, 5, 1999, pp. 2536-2555

[2] D. Giuli, A. Toccafondi, G. Biffi Gentili and A. Freni, "Tomographic Reconstruction of Rainfall Fields through Microwave Attenuation Measurements", J. Appl. Meteor., vol. 30, pp. 1323-1340, 1991

[3] F. Cuccoli, L. Facheris, S. Tanelli, D. Giuli: "Microwave attenuation measurements in satellite-ground links: spectral analysis for water vapor profile retrieval" submitted for publication on IEEE Trans. Geosc. Rem. Sens.

[4] F. Cuccoli, L. Facheris, S. Tanelli, D. Giuli "Infrared tomographic system for monitoring the two-dimensional distribution of atmospheric pollution over limited areas" IEEE Trans. Geosc. Rem. Sens.38, 1, 2000, pp. 155-168

[5] F. Cuccoli, L. Facheris, S. Tanelli, D. Giuli: "A feasibility study for active remote sensing of atmospheric carbon monoxide based on differential absorption of infrared radiation along vertical paths" submitted for publication on IEEE Trans. Geosc. Rem. Sens. 